\title{
STABILITY RESULTS FOR NONLINEAR FRACTIONAL ORDER $h$-DIFFERENCE SYSTEMS
}

\author{
XIANG LIU ${ }^{1}$, BAOGUO JIA ${ }^{2}$, LYNN ERBE $^{3}$, AND ALLAN PETERSON ${ }^{4}$ \\ ${ }^{1,2}$ School of Mathematics \\ Sun Yat-Sen University \\ Guangzhou, 510275, P.R. CHINA \\ ${ }^{3,4}$ Department of Mathematics \\ University of Nebraska-Lincoln \\ Lincoln, NE 68588-0130, USA
}

\begin{abstract}
This paper is concerned with the stability of the fractional order $h$-difference systems. The definition of Mittag-Leffler stability is introduced, and the sufficient conditions are presented by extending the Lyapunov direct method to such systems. Moreover, we weaken the restriction on Lyapunov function, the stability of the fractional order $h$-difference systems is established. Two numerical examples are given to illustrate our main results.
\end{abstract}

AMS Subject Classification: 39A11, 39A70

Key Words: fractional order $h$-difference systems, Lyapunov direct method, MittagLeffler stability, stability

Received: December 27, 2017; Accepted: July 11, 2018;

Published: July 20, 2018 doi: 10.12732/dsa.v27i3.10

Dynamic Publishers, Inc., Acad. Publishers, Ltd. https://acadsol.eu/dsa

\section{INTRODUCTION}

Fractional calculus is a field of mathematics that grows out of the traditional definitions of integral, derivatives, differences operators and deals with fractional integrals, derivatives and differences of any order. Basic information on fractional calculus, concepts, ideas and their applications can be found in $[1,2,3]$. This paper will focus on discrete fractional calculus that was initiated by Miller and Ross in [4], which are mainly about the calculus of the fractional $h$-differences, see $[5,7,8,9,10,11]$. Due to 
the wide application of the fractional calculus in science and engineering, it is becoming an interesting topic to investigate stability of the fractional systems. Recently, fractional calculus was introduced to the stability analysis of nonlinear systems, see $[12,13,14]$. The integer order methods of stabilization were used in these works. However, due to the memory effects of the fractional operators, it is not an easy task to directly extend the normal Lyapunov stability results to the fractional cases since the Leibniz law becomes complicated and cannot hold generally.

So, in order to prove the stability of the fractional order nonlinear systems, some other techniques must be applied. Matignon [15] proposes an explicit stability condition for a linear fractional differential systems. The articles $[16,17]$ propose the fractional Lyapunov direct method to the fractional order differential systems. In $[18,19,20]$, the authors have used this technique to obtain the Mittag-Leffler stability of the fractional order systems. However, there are few works on the fractional order $h$-difference systems.

In this paper, we give the definition of the Mittag-Leffler stability, and the sufficient conditions of the Mittag-Leffler stability by extending the Lyapunov direct method to the fractional order $h$-difference systems. Moreover, we weaken the restriction on Lyapunov function to obtain the stability of such systems. Finally, two numerical examples are given to illustrate our main results.

\section{PRELIMINARY DEFINITIONS}

Let $\mathcal{F}_{D}$ denote the set of real valued functions defined on $D$. We use the notation $(h \mathbb{N})_{a}:=\{a, a+h, a+2 h, \cdots\}$, where $h>0, a \in \mathbb{R}$. Let $\sigma(t)=t+h$ for $t \in(h \mathbb{N})_{a}$. For the convenience of the readers, we will list some relevant results here.

Definition 2.1. (See [5, Definition 2.1]). For a function $y \in \mathcal{F}_{(h \mathbb{N})_{a}}$, the forward $h$-difference operator is defined as

$$
\left(\Delta_{h} y\right)(t)=\frac{y(\sigma(t))-y(t)}{h}, \quad t \in(h \mathbb{N})_{a} .
$$

and the $h$-difference sum is given by

$$
\left({ }_{a} \Delta_{h}^{-1} y\right)(t)=\sum_{s=\frac{a}{h}}^{\frac{t}{h}-1} y(s h) h, \quad t \in(h \mathbb{N})_{a},
$$

where, by convention, $\left({ }_{a} \Delta_{h}^{-1} y\right)(a)=0$.

Definition 2.2. (See [10, Definition 2.6]). For arbitrary $t, \nu \in \mathbb{R}$, the $h$-factorial function is defined by

$$
t_{h}^{(\nu)}=h^{\nu} \frac{\Gamma\left(\frac{t}{h}+1\right)}{\Gamma\left(\frac{t}{h}+1-\nu\right)},
$$


where $\Gamma$ is the Euler gamma function with $\frac{t}{h}+1 \notin Z_{-} \cup\{0\}$, and we use the convention that $t_{h}^{(\nu)}=0$, when $\frac{t}{h}+1-\nu$ is a nonpositive integer, and $\frac{t}{h}+1$ is not a nonpositive integer.

Definition 2.3. (See [10, Definition 2.8]). For a function $y \in \mathcal{F}_{(h \mathbb{N})_{a}}$, the fractional $h$-sum of order $\nu>0$ is given by

$$
\left({ }_{a} \Delta_{h}^{-\nu} y\right)(t)=\frac{h}{\Gamma(\nu)} \sum_{s=\frac{a}{h}}^{\frac{t}{h}-\nu}(t-\sigma(s h))_{h}^{(\nu-1)} y(s h), \quad t \in(h \mathbb{N})_{a+\nu h},
$$

and $\left({ }_{a} \Delta_{h}^{0} y\right)(t)=y(t), \sigma(s h)=(s+1) h$.

Definition 2.4. (See [5, Definition 2.6]). Let $\nu \in(n-1, n]$, and $\mu=n-\nu$, where $n \in \mathbb{N}$. The Riemann-Liouville like fractional $h$-difference operator ${ }_{a} \Delta_{h}^{\nu} y$ of order $\nu$ for a function $y \in \mathcal{F}_{(h \mathbb{N})_{a}}$ is defined by

$$
\begin{aligned}
\left({ }_{a} \Delta_{h}^{\nu} y\right)(t)=\left(\Delta_{h}^{n}\left({ }_{a} \Delta_{h}^{-\mu} y\right)\right)(t)=\frac{h}{\Gamma(\mu)} \Delta_{h}^{n} \sum_{s=\frac{a}{h}}^{\frac{t}{h}-\mu}(t-\sigma(s h))_{h}^{(\mu-1)} y(s h) & \\
& t \in(h \mathbb{N})_{a+\mu h} .
\end{aligned}
$$

Lemma 2.1. (See [5, Theorem 2.8]). Let $\nu \in(n-1, n]$, and $\mu=n-\nu$, where $n \in \mathbb{N}$. The following formula is equivalent to (2.4):

$$
\begin{aligned}
& \left({ }_{a} \Delta_{h}^{\nu} y\right)(t) \\
& \quad=\left\{\begin{array}{l}
\frac{h}{\Gamma(-\nu)} \sum_{s=\frac{a}{h}}^{\frac{t}{h}+\nu}(t-\sigma(s h))_{h}^{(-\nu-1)} y(s h), \quad \nu \in(n-1, n), \quad t \in(h \mathbb{N})_{a+\mu h} \\
\left(\Delta_{h}^{n} y\right)(t), \quad \nu=n, t \in(h \mathbb{N})_{a} .
\end{array}\right.
\end{aligned}
$$

Lemma 2.2. (See [5, Definition 2.9]). Let $\nu \in(n-1, n]$, and $\mu=n-\nu$, where $n \in \mathbb{N}$. The Caputo like h-difference operator ${ }_{a} \Delta_{h, *}^{\nu}$ of order $\nu$ for a function $y \in \mathcal{F}_{(h \mathbb{N})_{a}}$ is defined by

$$
\begin{aligned}
&\left({ }_{a} \Delta_{h, *}^{\nu} y\right)(t)=\left({ }_{a} \Delta_{h}^{-\mu}\left(\Delta_{h}^{n} y\right)\right)(t) \\
&=\frac{h}{\Gamma(\mu)} \sum_{s=\frac{a}{h}}^{\frac{t}{h}-\mu}(t-\sigma(s h))_{h}^{(\mu-1)}\left(\Delta_{h}^{n} y\right)(s h), \quad t \in(h \mathbb{N})_{a+\mu h} .
\end{aligned}
$$

Lemma 2.3. (See [5, Proposition 2.11]). Let $\nu \in(n-1, n]$, and $\mu=n-\nu$, where 
$n \in \mathbb{N}$. The following formula is equivalent to (2.6):

$$
\left({ }_{a} \Delta_{h, *}^{\nu} y\right)(t)=\left\{\begin{array}{l}
\frac{h^{1-n}}{\Gamma(n-\nu)} \sum_{s=\frac{a}{h}}^{\frac{t}{h}-(n-\nu)}(t-\sigma(s h))_{h}^{(n-\nu-1)} \sum_{r=0}^{n}(-1)^{r+1}\left(\begin{array}{c}
n \\
r
\end{array}\right) y((r+s) h), \\
\left(\Delta_{h}^{n} y\right)(t), \quad \nu=n, t \in(h \mathbb{N})_{a} .
\end{array}\right.
$$

Property 2.1. The following properties are useful in this paper:

Delta difference of the h-falling factorial function (See [7, Lemma 3.2])

$$
{ }_{s} \Delta_{h}(t-s h)_{h}^{(\nu)}=-\nu(t-\sigma(s h))_{h}^{(\nu-1)},
$$

where ${ }_{s} \Delta_{h}(t-s h)_{h}^{(\nu)}=\frac{(t-s h-h)_{h}^{(\nu)}-(t-s h)_{h}^{(\nu)}}{h}$.

Definition 2.5. (See $[11$, Definition 7]). For $\lambda \in \mathbb{R}$, and $\alpha, \beta, z \in \mathbb{C}$ with $\operatorname{Re}(\alpha)>0$, the discrete Mittag-Leffler functions are defined by

$$
\mathcal{E}_{(\alpha, \beta)}(\lambda, z)=\sum_{k=0}^{\infty} \lambda^{k} \frac{(z+(k-1)(\alpha-1))^{(k \alpha)}(z+k(\alpha-1))^{(\beta-1)}}{\Gamma(\alpha k+\beta)} .
$$

For $\beta=1$, we write $E_{(\alpha)}=E_{(\alpha, 1)}$. The following Mittag-Leffler functions are defined in a different manner, but both definitions give the same values.

$$
E_{(\alpha, \beta)}(\lambda, n)=\sum_{k=0}^{\infty} \lambda^{k} \widetilde{\varphi}_{k \alpha+\beta}(n-k)=\sum_{k=0}^{n} \lambda^{k} \widetilde{\varphi}_{k \alpha+\beta}(n-k),
$$

where

$$
\widetilde{\varphi}_{\alpha}(n)=\left(\begin{array}{c}
n+\alpha-1 \\
n
\end{array}\right)=(-1)^{n}\left(\begin{array}{c}
-\alpha \\
n
\end{array}\right)
$$

Now, let us recall that the $\mathcal{Z}$-transform of a sequence $\{y(n)\}_{n \in \mathbb{N}_{0}}$ is a complex function given by $Y(z)=\mathcal{Z}[y](z)=\sum_{k=0}^{\infty} \frac{y(k)}{z^{k}}$, where $z \in \mathbb{C}$ is a complex number for which this series converges absolutely. The $\mathcal{Z}$-transform of $\widetilde{\varphi}_{\alpha}$ is given by

$$
\mathcal{Z}\left[\widetilde{\varphi}_{\alpha}\right](z)=\sum_{k=0}^{\infty}\left(\begin{array}{c}
k+\alpha-1 \\
k
\end{array}\right) \frac{1}{z^{k}}=\sum_{k=0}^{\infty}(-1)^{k}\left(\begin{array}{c}
-\alpha \\
k
\end{array}\right) \frac{1}{z^{k}}=\left(\frac{z}{z-1}\right)^{\alpha} .
$$

The convolution of $\tilde{\varphi}_{\alpha}$ and $x$ is defined as

$$
\left(\tilde{\varphi}_{\alpha} * x\right)(n)=\sum_{s=0}^{n}\left(\begin{array}{c}
n-s+\alpha-1 \\
n-s
\end{array}\right) x(s) .
$$

The following relations hold for the $\mathcal{Z}$-transform,

$$
\mathcal{Z}[x(n) * y(n)](z)=\mathcal{Z}[x(n)](z) \mathcal{Z}[y(n)](z),
$$




$$
\begin{gathered}
\mathcal{Z}[y(n-1)](z)=\frac{1}{z} \mathcal{Z}[y(n)](z), \\
\mathcal{Z}[y(n+1)](z)=z \mathcal{Z}[y(n)](z)-z y(0)
\end{gathered}
$$

for $|z|>R$, where $R$ is the radius of convergence of $\mathcal{Z}[y(n)](z)$.

Proposition 2.1. (See [5, Proposition 4.5]). Let $\nu \in(0,1], h>0, a=(\nu-1) h$, and $y$ be a real-valued function defined on $(h \mathbb{N})_{a}$. Then the following formula holds

$$
\left({ }_{0} \Delta_{h}^{-\nu}\left({ }_{a} \Delta_{h, *}^{\nu} y\right)\right)(t)=y(t)-y(a), \quad t \in(h \mathbb{N})_{\nu h} .
$$

The following corollary appears in Mozyrska et al [5, Corollary 4.2].

Corollary 2.1. (See [5, Corollary 4.2]). Let $\nu \in(0,1]$, one can get

$$
\left({ }_{a} \Delta_{h, *}^{\nu} y\right)(t)=\left({ }_{a} \Delta_{h}^{\nu} y\right)(t)-\frac{(t-a)_{h}^{(-\nu)} y(a)}{\Gamma(1-\nu)}, \quad t \in(h \mathbb{N})_{a+(1-\nu) h} .
$$

Proposition 2.2. (See [11, Proposition 8]). Let $E_{\alpha, \beta}(\lambda, \cdot)$ be defined by (2.10). Then

$$
\mathcal{Z}\left[E_{(\alpha, \beta)}(\lambda, \cdot)\right](z)=\left(\frac{z}{z-1}\right)^{\beta}\left(1-\frac{\lambda}{z}\left(\frac{z}{z-1}\right)^{\alpha}\right)^{-1},
$$

where $|z|>1$ and $|z-1|^{\alpha}|z|^{1-\alpha}>|\lambda|$.

Proposition 2.3. (See [5, Proposition 5.3]). Let $\nu \in(0,1]$, and $a=(\nu-1) h$. The linear initial value problem (IVP)

$$
\left\{\begin{array}{l}
\left({ }_{a} \Delta_{h, *}^{\nu} y\right)(t)=-\lambda y(t+a), \quad t \in(h \mathbb{N})_{0} \\
y(a)=y_{0}, \quad y_{0} \in \mathbb{R}
\end{array}\right.
$$

has a unique solution given by the formula

$$
y(t)=\mathcal{E}_{(\nu)}\left(-\lambda h^{\nu}, \frac{t}{h}\right) y_{0}=E_{(\nu)}\left(-\lambda h^{\nu}, n\right) y_{0},
$$

where $t \in(h \mathbb{N})_{a}$, and $\frac{t}{h}=n+\nu-1 \in \mathbb{N}_{\nu-1}$.

Next, we will give some lemmas concerning the properties of solutions for the IVP (2.12).

Lemma 2.4. Assume $y(t)$ is a solution of the $\operatorname{IVP}(2.12), y(a)>0$, and $0<\lambda \leq$ $\nu h^{-\nu}$. Then $\left(\Delta_{h} y\right)(t)<0$ for $t \in(h \mathbb{N})_{a}$.

Proof. Apply the operator $\Delta_{h}$ to both sides of the first equality of the IVP (2.12), we have

$$
\left({ }_{a} \Delta_{h}^{\nu}\left(\Delta_{h} y\right)\right)(t)=-\lambda\left(\Delta_{h} y\right)(t+\nu h-h),
$$


that is,

$$
\frac{h}{\Gamma(-\nu)} \sum_{s=\frac{a}{h}}^{\frac{t}{h}+\nu}(t-\sigma(s h))_{h}^{(-\nu-1)}\left(\Delta_{h} y\right)(s h)=-\lambda\left(\Delta_{h} y\right)(t+\nu h-h),
$$

which can be written as,

$$
\begin{aligned}
& \frac{h}{\Gamma(-\nu)} \sum_{s=\frac{a}{h}}^{\frac{t}{h}+\nu-2}(t-\sigma(s h))_{h}^{(-\nu-1)}\left(\Delta_{h} y\right)(s h) \\
& \quad=\left(\nu h^{-\nu}-\lambda\right)\left(\Delta_{h} y\right)(t+\nu h-h)-h^{-\nu}\left(\Delta_{h} y\right)(t+\nu h) .
\end{aligned}
$$

Arguing by contradiction, we assume that there exists a first point $t_{1}$ such that $\left(\Delta_{h} y\right)(t) \geq 0$ on $\left[t_{1}, t_{2}\right] \cap(h \mathbb{N})_{a}$, where $t_{1}, t_{2} \in(h \mathbb{N})_{a+h}$, and $\left(\Delta_{h} y\right)(t)<0$ on $\left[a, t_{1}-h\right] \cap(h \mathbb{N})_{a}$. Let $t_{2}^{*}=t_{2}-\nu h, t_{1}^{*}=t_{1}-\nu h$, then from the equation (2.13), we have

$$
\begin{aligned}
\frac{h}{\Gamma(-\nu)} \sum_{s=\frac{a}{h}}^{\frac{t_{1}^{*}}{h}+\nu-2}\left(t_{1}^{*}-\right. & \sigma(s h))_{h}^{(-\nu-1)}\left(\Delta_{h} y\right)(s h) \\
& =\left(\nu h^{-\nu}-\lambda\right)\left(\Delta_{h} y\right)\left(t_{1}^{*}+\nu h-h\right)-h^{-\nu}\left(\Delta_{h} y\right)\left(t_{1}^{*}+\nu h\right) .
\end{aligned}
$$

we can see that the LHS of the equation (2.14) is greater than zero, while the RHS of the equation $(2.14)$ is less than or equal to zero, which is a contradiction. So, we have $\left(\Delta_{h} y\right)(t)<0$ for $t \in(h \mathbb{N})_{a}$. The proof is complete.

Lemma 2.5. Assume $\lambda>0$, and $y(a)>0$. Then the solution of the IVP (2.12) satisfies $y(t)>0$ for $t \in(h \mathbb{N})_{a}$.

Proof. From $\left(\Delta_{h} y\right)(t)<0$, and Lemma 2.2, we can obtain

$$
\left({ }_{a} \Delta_{h, *}^{\nu} y\right)(t)<0, \quad \nu \in(0,1], \quad t \in(h \mathbb{N})_{0} .
$$

As a result, we can get $y(t)>0$ by the first equality of the IVP (2.12). The proof is complete.

Lemma 2.6. Assume $\lambda>0$, and $y(a)>0$. Then

$$
\lim _{t \rightarrow \infty} \mathcal{E}_{(\nu)}\left(-\lambda h^{\nu}, \frac{t}{h}\right)=0
$$

Proof. By Lemma 2.4, 2.5, we can see the limit exists. Arguing by contradiction, we assume $\lim _{t \rightarrow \infty} y(t)=c>0$ for $t \in(h \mathbb{N})_{a}$. Apply the operator ${ }_{0} \Delta_{h}^{-\nu}$ to both sides of the first equality of the IVP (2.12), we have

$$
\left({ }_{0} \Delta_{h}^{-\nu}\left({ }_{a} \Delta_{h, *}^{\nu} y\right)\right)(t)=-\lambda\left({ }_{0} \Delta_{h}^{-\nu} y\right)(t+a) .
$$


By Proposition 2.1, we have

$$
\begin{aligned}
y(t)-y(a) & =-\frac{\lambda h}{\Gamma(\nu)} \sum_{s=\frac{0}{h}}^{\frac{t}{h}-\nu}(t-\sigma(s h))_{h}^{(\nu-1)} y(a+s h) \\
& \leq-\frac{\lambda h y(t)}{\Gamma(\nu)} \sum_{s=\frac{0}{h}}^{\frac{t}{h}-\nu}(t-\sigma(s h))_{h}^{(\nu-1)} \\
& =-\frac{\lambda y(t) t_{h}^{(\nu)}}{\Gamma(\nu+1)} .
\end{aligned}
$$

Note that

$$
\lim _{t \rightarrow \infty}(y(t)-y(a))=c-y(a)<0 .
$$

On the other hand, we have

$$
\lim _{t \rightarrow \infty}-\frac{\lambda y(t) t_{h}^{(\nu)}}{\Gamma(\nu+1)}=-\infty
$$

which is a contradiction. So, we have

$$
\lim _{t \rightarrow \infty} \mathcal{E}_{(\nu)}\left(-\lambda h^{\nu}, \frac{t}{h}\right)=0 .
$$

The proof is complete.

\section{MAIN RESULTS}

In this section, we will introduce the stability notions for the fractional order $h$ difference systems involving both the Caputo and the Riemann-Liouville type operators.

Consider the following vector initial value problems (IVPS)

$$
\left\{\begin{array}{l}
\left({ }_{a} \Delta_{h, *}^{\nu} x\right)(t)=f(t, x(t+\nu h-h)), \quad t \in(h \mathbb{N})_{0}, \\
x(a)=x_{0} \in \mathbb{R}^{n}
\end{array}\right.
$$

and

$$
\left\{\begin{array}{l}
\left({ }_{a} \Delta_{h}^{\nu} x\right)(t)=f(t, x(t+\nu h-h)), \quad t \in(h \mathbb{N})_{0}, \\
x(a)=x_{0} \in \mathbb{R}^{n}
\end{array}\right.
$$

where $f:(h \mathbb{N})_{0} \times \mathbb{R}^{n} \rightarrow \mathbb{R}^{n}, x:(h \mathbb{N})_{a} \rightarrow \mathbb{R}^{n}, a=(\nu-1) h \in(-h, 0] \subset \mathbb{R}$, and $\nu \in(0,1]$. It is easy to see that every IVP (3.1) and (3.2) has a unique solution which exists in $(h \mathbb{N})_{a}$.

The constant vector $x_{e q}$ is an equilibrium point of the vector fractional order $h$ difference equation (3.1) (or (3.2)) if and only if $\left({ }_{a} \Delta_{h, *}^{\nu} x_{e q}\right)(t)=f\left(t, x_{e q}(t+\nu h-h)\right)=$ 
$0\left(\left({ }_{a} \Delta_{h}^{\nu} x_{e q}\right)(t)=f\left(t, x_{e q}(t+\nu h-h)\right)\right.$ in the case of the Riemann-Liouville vector fractional order $h$-difference equation) for all $t \in(h \mathbb{N})_{0}$.

Assume that $f(t, 0)=0$ so that the trivial solution $x \equiv 0$ is an equilibrium point of the fractional order $h$-difference system (3.1) (or (3.2)). Note that there is no loss of generality in doing so because any equilibrium point can be shifted to the origin via a change of variables.

First, we present the following simple lemmas and important facts.

Lemma 3.1. Let $\nu \in(0,1]$. Then $\left\|\left({ }_{0} \Delta_{h}^{-\nu} f\right)(t, x(t+\nu h-h))\right\| \leq\left({ }_{0} \Delta_{h}^{-\nu}\|f\|\right)(t, x(t+$ $\nu h-h))$ holds for $t \in(h \mathbb{N})_{\nu h}$.

Proof. According to Definition 2.3, we have

$$
\begin{aligned}
& \left\|\left({ }_{0} \Delta_{h}^{-\nu} f\right)(t, x(t+\nu h-h))\right\| \\
& =\left\|\frac{h}{\Gamma(\nu)} \sum_{s=\frac{0}{h}}^{\frac{t}{h}-\nu}(t-\sigma(s h))_{h}^{(\nu-1)} f(s h, x(s h+\nu h-h))\right\| \\
& \quad \leq \frac{h}{\Gamma(\nu)} \sum_{s=\frac{0}{h}}^{\frac{t}{h}-\nu}(t-\sigma(s h))_{h}^{(\nu-1)}\|f(s h, x(s h+\nu h-h))\| \\
& \quad \leq\left({ }_{0} \Delta_{h}^{-\nu}\|f\|\right)(t, x(t+\nu h-h)) .
\end{aligned}
$$

The proof is complete.

Proposition 3.1. (See [11, Proposition 4]). For $a \in \mathbb{R}, \nu \in(0,1]$, let $y(n):=$ $\left({ }_{a} \Delta_{h}^{-\nu} x\right)(t)$, where $t \in(h \mathbb{N})_{a+\nu h}$ and $t=a+\nu h+n h$. Then

$$
\mathcal{Z}[y](z)=\left(\frac{h z}{z-1}\right)^{\nu} X(z)
$$

where $X(z)=\mathcal{Z}[\bar{x}](z), \bar{x}(n)=x(a+n h)$.

Proposition 3.2. (See [11, Proposition 16]). For $a \in \mathbb{R}, \nu \in(0,1]$, let $y(n):=$ $\left({ }_{a} \Delta_{h, *}^{\nu} x\right)(t)$, where $t \in(h \mathbb{N})_{a+(1-\nu) h}$, and $t=a+(1-\nu) h+n h$. Then

$$
\mathcal{Z}[y](z)=h^{-\nu}\left(\frac{z}{z-1}\right)^{1-\nu}[(z-1) X(z)-z x(a)]
$$

where $X(z)=\mathcal{Z}[\bar{x}](z)$, and $\bar{x}(n)=x(a+n h)$.

Proposition 3.3. (See [11, Proposition 24]). For $a \in \mathbb{R}, \nu \in(0,1]$, let $y(n):=$ $\left({ }_{a} \Delta_{h}^{\nu} x\right)(t)$, where $t \in(h \mathbb{N})_{a+(1-\nu) h}$, and $t=a+(1-\nu) h+n h$. Then

$$
\mathcal{Z}[y](z)=z\left(\frac{h z}{z-1}\right)^{-\nu} X(z)-z h^{-\nu} x(a)
$$

where $X(z)=\mathcal{Z}[\bar{x}](z)$, and $\bar{x}(n)=x(a+n h)$. 
In the following, we study the relationship between the Lipschitz condition and the Caputo fractional order $h$-difference system (3.1).

Theorem 3.1. Assume $x=0$ is an equilibrium point of the system (3.1), and $f$ is Lipschitz in $x$ with Lipschitz constant L. Then the solution of the system (3.1) satisfies

$$
\|x(a+n h)\| \leq E_{(\nu)}\left(L h^{\nu}, n\right)\left\|x_{0}\right\|, \quad \nu \in(0,1]
$$

Proof. Set $\bar{f}(n)=f(n h, x(a+n h))$, and $\bar{x}(n)=x(a+n h)$ for $n \in \mathbb{N}_{0}$. Applying the operator ${ }_{0} \Delta_{h}^{-\nu}$ to both sides of the first equality of the system (3.1), and the Proposition 2.1, we have

$$
x(\nu h+n h)-x(a)=\left({ }_{0} \Delta_{h}^{-\nu} f\right)(\nu h+n h, x(a+n h+\nu h)),
$$

which can be written as

$$
x(a+n h+h)-x_{0}=\left({ }_{0} \Delta_{h}^{-\nu} f\right)(\nu h+n h, x(a+n h+\nu h)) .
$$

Using the Lipschitz condition, we obtain

$$
\begin{aligned}
\left|\|\bar{x}(n+1)\|-\left\|x_{0}\right\|\right| \leq\left\|\bar{x}(n+1)-x_{0}\right\| & =\left\|\left({ }_{0} \Delta_{h}^{-\nu} \bar{f}\right)(n+\nu)\right\| \\
& \leq\left({ }_{0} \Delta_{h}^{-\nu}\|\bar{f}\|\right)(n+\nu) \\
& \leq L\left({ }_{0} \Delta_{h}^{-\nu}\|\bar{x}\|\right)(n+\nu) .
\end{aligned}
$$

There exists a nonnegative function $M(\cdot)$ satisfying

$$
\|\bar{x}(n+1)\|-\left\|x_{0}\right\|=L\left({ }_{0} \Delta_{h}^{-\nu}\|\bar{x}\|\right)(n+\nu)-M(n) .
$$

Using $\mathcal{Z}$-transform for the equation (3.7), and Proposition 3.1, we receive that

$$
z\|X(z)\|-z\left\|x_{0}\right\|-\frac{z}{z-1}\left\|x_{0}\right\|=L\left(\frac{h z}{z-1}\right)^{\nu}\|X(z)\|-M(z) .
$$

Then, we have

$$
\|X(z)\|=\frac{z}{z-1} W(z)\left\|x_{0}\right\|-\frac{1}{z} W(z) M(z)
$$

where $\|X(z)\|=\mathcal{Z}[\|\bar{x}\|](z), M(z)=\mathcal{Z}[M](z), W(z)=\left[1-L \frac{1}{z}\left(\frac{h z}{z-1}\right)^{\nu}\right]^{-1}$. Applying the inverse $\mathcal{Z}$-transform to the equation (3.8), we get

$$
\|\bar{x}(n)\|=E_{(\nu)}\left(L h^{\nu}, n\right)\left\|x_{0}\right\|-\left(E_{(\nu, 0)}\left(L h^{\nu}, \cdot\right) * M\right)(n-1) .
$$

And as for $L>0, M(\cdot) \geq 0$, also: $\left(E_{(\nu, 0)}\left(L h^{\nu}, \cdot\right) * M\right)(n-1) \geq 0$, then, we have

$$
\|\bar{x}(n)\| \leq E_{(\nu)}\left(L h^{\nu}, n\right)\left\|x_{0}\right\| .
$$

The proof is complete. 
Remark 3.1. In Theorem 3.1, if $\nu=1$, it follows from (2.10) that

$$
\|\bar{x}(n)\| \leq(1+L h)^{n}\left\|x_{0}\right\|
$$

Definition 3.1. The equilibrium point $x=0$ of the system (3.1) (or (3.2)) is said to be

(a) stable, if for each $\varepsilon>0$, there exists $\delta=\delta(\varepsilon)>0$ such that $\|x(a)\|<\delta$ implies $\|x(a+k h)\|<\varepsilon$ for all $k \in \mathbb{N}_{0}$.

(b) attractive, if there exists $\delta>0$ such that $\|x(a)\|<\delta$ implies $\lim _{k \rightarrow \infty} x(a+$ $k h)=0$.

(c) asymptotically stable, if it is stable and attractive.

The fractional difference system (3.1) (or (3.2)) is called stable (asymptotically stable) if their equilibrium point $x=0$ is stable (asymptotically stable).

Definition 3.2. (See [21, Definition 3.2]). A function $\phi(r)$ is said to belong to the class $\mathcal{K}$ if and only if $\phi \in C\left[[0, \rho), \mathbb{R}_{+}\right], \phi(0)=0$, and $\phi(r)$ is strictly monotonically increasing in $r$.

Now, we define the stability in the sense of Mittag-Leffler.

Definition 3.3. The solutions of the system (3.1) (or (3.2)) is said to be MittagLeffler stable if

$$
\|x(a+n h)\| \leq\left\{m\left(x_{0}\right) E_{(\nu)}\left(-\lambda h^{\nu}, n\right)\right\}^{b},
$$

where $\nu \in(0,1), \lambda>0, b>0, m(0)=0, m(x) \geq 0$, and $m$ is locally Lipschitz for $x \in B \in \mathbb{R}^{n}$ with Lipschiz constant $m_{0}$.

Lemma 3.2. Mittag-Leffler stability implies asymptotic stability.

Proof. We want to show that for every $\varepsilon$, there exists a $\delta$, such that for every $x(a)=x_{0}<\delta$, we have $\|x(a+k h)\|<\varepsilon$. Since we have

$$
\|x(a+n h)\| \leq\left\{m\left(x_{0}\right) E_{(\nu)}\left(-\lambda h^{\nu}, n\right)\right\}^{b} .
$$

For $\nu \in(0,1)$, we have

$$
E_{(\nu)}\left(-\lambda h^{\nu}, n\right) \leq 1
$$

Hence

$$
m^{b}\left(x_{0}\right)\left(E_{(\nu)}\left(-\lambda h^{\nu}, n\right)\right)^{b} \leq m^{b}\left(x_{0}\right)
$$

Therefore, we have

$$
\|x(a+n h)\| \leq m^{b}\left(x_{0}\right) .
$$


Let $\Omega$ be a compact ball, $\Omega_{r}=\left\{x \in R^{n}:\|x\| \leq r\right\}$, where $r$ is a given radius. Since $m(x)$ is Lipschitz for $x$, then $m$ is continuous, and $m(x)$ achieves its maximal and minimal values on $\Omega_{r}$. Then for every $\left\|x_{0}\right\|<r$, we have

$$
\|x(a+n h)\|<m^{b}\left(x_{0}\right)<\max m^{b}\left(\Omega_{r}\right)=\varepsilon,
$$

Hence, the origin is stable.

To prove the asymptotic stability, it is sufficient to show the attractivity of the origin. It can be done directly from the definition of Mittag-Leffler stability, we have

$$
\|x(a+n h)\| \leq\left\{m\left(x_{0}\right) E_{(\nu)}\left(-\lambda h^{\nu}, n\right)\right\}^{b},
$$

where $m\left(x_{0}\right)$ has a finite value. From Lemma 2.6, we have $E_{(\nu)}\left(-\lambda h^{\nu}, n\right) \rightarrow 0$ as $n \rightarrow \infty$. Thus, $\lim _{n \rightarrow \infty}\|x(a+n h)\|=0$. Therefore, the origin is asymptotically stable. The proof is complete.

Now, we give the following comparison result, which is important for our main results.

Lemma 3.3. Assume $\left({ }_{a} \Delta_{h, *}^{\nu} u\right)(t) \geq\left({ }_{a} \Delta_{h, *}^{\nu} v\right)(t), t \in(h \mathbb{N})_{0}, u(a)=v(a)$, and $\nu \in(0,1]$. Then we have $u(t) \geq v(t)$ for $t \in(h \mathbb{N})_{a}$.

Proof. It follows from $\left({ }_{a} \Delta_{h, *}^{\nu} u\right)(t) \geq\left({ }_{a} \Delta_{h, *}^{\nu} v\right)(t)$ that there exists a nonnegative function $M(\cdot)$ satisfying

$$
\left({ }_{a} \Delta_{h, *}^{\nu} u\right)(t)=\left({ }_{a} \Delta_{h, *}^{\nu} v\right)(t)+M(t) .
$$

Taking the $\mathcal{Z}$-transform of the equation (3.9), and using the Proposition 3.2, we get $h^{-\nu}\left(\frac{z}{z-1}\right)^{1-\nu}[(z-1) U(z)-z u(a)]=h^{-\nu}\left(\frac{z}{z-1}\right)^{1-\nu}[(z-1) V(z)-z v(a)]+M(z)$, where $U(z)=\mathcal{Z}[u](z), V(z)=\mathcal{Z}[v](z)$. It follows from $u(a)=v(a)$ that

$$
U(z)=V(z)+h^{\nu} \frac{1}{z}\left(\frac{z}{z-1}\right)^{\nu} M(z) .
$$

Applying the inverse $\mathcal{Z}$-transform to the equation (3.10), we obtain

$$
u(t)=v(t)+h^{\nu}\left(\widetilde{\varphi}_{\nu} * M\right)(n-1) .
$$

It follows from $h^{\nu}\left(\widetilde{\varphi}_{\nu} * M\right)(n-1) \geq 0$ that $u(t) \geq v(t)$. The proof is complete.

In the following, we extend the Lyapunov direct method to the fractional order $h$-difference systems, which leads to the Mittag-Leffler stability. Let $\bar{V}: \mathbb{N}_{0} \rightarrow \mathbb{R}$, and $\bar{V}(n)=V(n, x(a+n h))$ for $n \in \mathbb{N}_{0}$. 
Theorem 3.2. Assume $x=0$ be an equilibrium point of the system (3.1) and $\mathcal{D} \subseteq \mathbb{R}^{n}$ be a domain containing the origin. Let $V: \mathbb{N}_{0} \times \mathcal{D} \rightarrow \mathbb{R}$ be a function that is Lipschitz with respect to $x$ and such that

$$
\begin{gathered}
\alpha_{1}\|x\|^{a} \leq \bar{V}(n) \leq \alpha_{2}\|x\|^{a b}, \\
\left({ }_{0} \Delta_{h, *}^{\beta} \bar{V}\right)(n) \leq-\alpha_{3}\|x\|^{a b},
\end{gathered}
$$

where $n \in \mathbb{N}_{0}, x \in \mathcal{D}, \beta \in(0,1), \alpha_{1}, \alpha_{2}, \alpha_{3}, a$ and $b$ are arbitrary positive constants. Then $x=0$ is Mittag-Leffler stable.

Proof. It follows from the inequalities (3.11) and (3.12) that $\left({ }_{0} \Delta_{h, *}^{\beta} \bar{V}\right)(n) \leq-\frac{\alpha_{3}}{\alpha_{2}} \bar{V}(n)$. There exists a nonnegative function $M(\cdot)$ satisfying

$$
\left({ }_{0} \Delta_{h, *}^{\beta} \bar{V}\right)(n)+M(n)=-\frac{\alpha_{3}}{\alpha_{2}} \bar{V}(n) .
$$

Taking $Z$-transform of the equation (3.13), and Proposition 3.2, we get

$$
h^{-\beta}\left(\frac{z}{z-1}\right)^{1-\beta}[(z-1) V(z)-z \bar{V}(0)]+M(z)=-\frac{\alpha_{3}}{\alpha_{2}} V(z),
$$

where $\bar{V}(0)=V(0, x(a)), V(z)=\mathcal{Z}[\bar{V}](z), M(z)=\mathcal{Z}[M](z)$. Then it follows that

$$
V(z)=\frac{\frac{z}{z-1} \bar{V}(0)}{1+h^{\beta} \frac{\alpha_{3}}{\alpha_{2}} \frac{1}{z}\left(\frac{z}{z-1}\right)^{\beta}}-\frac{h^{\beta} \frac{1}{z}\left(\frac{z}{z-1}\right)^{\beta} M(z)}{1+h^{\beta} \frac{\alpha_{3}}{\alpha_{2}} \frac{1}{z}\left(\frac{z}{z-1}\right)^{\beta}} .
$$

If $x(a)=0$, namely $\bar{V}(0)=0$, then the solution of the system (3.1) is $x=0$. If $x(a) \neq 0$, then $\bar{V}(0)>0$. Then taking the inverse $\mathcal{Z}$-transform, we get that the solution of the equation (3.14) is as follows:

$$
\bar{V}(n)=E_{(\beta)}\left(-\frac{\alpha_{3}}{\alpha_{2}} h^{\beta}, n\right) \bar{V}(0)-h^{\beta}\left(E_{(\beta, \beta)}\left(-\frac{\alpha_{3}}{\alpha_{2}} h^{\beta}, \cdot\right) * M\right)(n-1) .
$$

Since $h^{\beta}\left(E_{(\beta, \beta)}\left(-\frac{\alpha_{3}}{\alpha_{2}} h^{\beta}, \cdot\right) * M\right)(n-1) \geq 0$, it follows that

$$
\bar{V}(n) \leq E_{(\beta)}\left(-\frac{\alpha_{3}}{\alpha_{2}} h^{\beta}, n\right) \bar{V}(0)
$$

Then substituting this inequality into the inequality (3.11), we get

$$
\|x(a+n h)\| \leq\left[\frac{\bar{V}(0)}{\alpha_{1}} E_{(\beta)}\left(-\frac{\alpha_{3}}{\alpha_{2}} h^{\beta}, n\right)\right]^{\frac{1}{a}},
$$

where $\frac{\bar{V}(0)}{\alpha_{1}}>0$ for $x(a) \neq 0$. Let $m=\frac{\bar{V}(0)}{\alpha_{1}}=\frac{V(0, x(a))}{\alpha_{1}} \geq 0$, then we have

$$
\|x(a+n h)\| \leq\left[m E_{(\beta)}\left(-\frac{\alpha_{3}}{\alpha_{2}} h^{\beta}, n\right)\right]^{\frac{1}{a}},
$$

where $m=0$ if and only if $x(a)=0$. Since $V$ is locally Lipschitz with respect to $x$ and $V(0, x(a))=0$ if and only if $x(a)=0$, it follows that $m=\frac{V(0, x(a))}{\alpha_{1}}$ is also Lipschitz with respect to $x(a)$ and $m(0)=0$, which implies the Mittag-Leffler stability of the system (3.1). The proof is complete. 
In fact, we can weaken the restriction on the function $\bar{V}(n)$, the following lemma will show this fact.

Theorem 3.3. Assume that $x=0$ be an equilibrium point of the system (3.1), $\mathcal{D} \subseteq \mathbb{R}^{n}$ be a domain containing the origin, and there exist a function $V: \mathbb{N}_{0} \times \mathcal{D} \rightarrow \mathbb{R}$, and class $\mathcal{K}$-function $\alpha$ satisfying

$$
\begin{gathered}
\bar{V}(n) \geq \alpha(\|x\|), \\
\left({ }_{0} \Delta_{h, *}^{\beta} \bar{V}\right)(n) \leq 0,
\end{gathered}
$$

where $n \in \mathbb{N}_{0}, x \in \mathcal{D}, \beta \in(0,1)$. Then the equilibrium point $x=0$ is stable.

Proof. It follows from Lemma 3.3, and the inequality $(3.17)$ that $\bar{V}(n) \leq \bar{V}(0)$. Again taking into account the inequality (3.16), we have

$$
\|x\| \leq \alpha^{-1}(\bar{V}(0))=\alpha^{-1}\left(V\left(0, x_{0}\right)\right) .
$$

According to the definition of stability, the equilibrium point $x=0$ is stable. The proof is complete.

For the convenience of readers, we give a relationship between the Caputo and the Riemann-Liouville type operators.

Lemma 3.4. Assume that $\nu \in(0,1]$, and $y(a) \geq 0$. Then $\left({ }_{a} \Delta_{h, *}^{\nu} y\right)(t) \leq\left({ }_{a} \Delta_{h}^{\nu} y\right)(t)$ for $t \in(h \mathbb{N})_{0}$.

Proof. Note that for $\nu=1$, we have the equality. Now, we consider the case for $\nu \in(0,1)$. From Corollary 2.1, we get

$$
\left({ }_{a} \Delta_{h, *}^{\nu} y\right)(a+n h+(1-\nu) h)=\left({ }_{a} \Delta_{h}^{\nu} y\right)(a+n h+(1-\nu) h)-\frac{(n h+(1-\nu) h)_{h}^{(-\nu)} y(a)}{\Gamma(1-\nu)}
$$

for $\nu \in(0,1), n \in \mathbb{N}_{0}$. Since $y(a) \geq 0$, and $(n h+(1-\nu) h)_{h}^{(-\nu)}>0$, then we have

$$
\left({ }_{a} \Delta_{h, *}^{\nu} y\right)(a+n h+(1-\nu) h) \leq\left({ }_{a} \Delta_{h}^{\nu} y\right)(a+n h+(1-\nu) h)
$$

for $n \in \mathbb{N}_{0}$. The proof is complete.

Lemma 3.5. Assume that $\left({ }_{a} \Delta_{h}^{\nu} u\right)(t) \geq\left({ }_{a} \Delta_{h}^{\nu} v\right)(t), t \in(h \mathbb{N})_{0}, u(a)=v(a)$, and $\nu \in(0,1]$. Then we have $u(t) \geq v(t)$ for $t \in(h \mathbb{N})_{a}$.

Proof. It follows from $\left({ }_{a} \Delta_{h}^{\nu} u\right)(t) \geq\left({ }_{a} \Delta_{h}^{\nu} v\right)(t)$ that there exists a nonnegative function $M(\cdot)$ satisfying

$$
\left({ }_{a} \Delta_{h}^{\nu} u\right)(t)=\left({ }_{a} \Delta_{h}^{\nu} v\right)(t)+M(t)
$$


Taking the $\mathcal{Z}$-transform of the equation (3.18), and using the Proposition 3.3, we get

$$
z\left(\frac{h z}{z-1}\right)^{-\nu} U(z)-z h^{-\nu} u(a)=h^{-\nu} z\left(\frac{h z}{z-1}\right)^{-\nu} V(z)-z h^{-\nu} v(a)+M(z) .
$$

It follows from $u(a)=v(a)$ that

$$
U(z)=V(z)+\frac{1}{z}\left(\frac{h z}{z-1}\right)^{\nu} M(z) .
$$

Applying the inverse $\mathcal{Z}$-transform to the equation (3.19), we have

$$
u(t)=v(t)+h^{\nu}\left(\widetilde{\varphi}_{\nu} * M\right)(n-1) .
$$

It follows from $h^{\nu}\left(\widetilde{\varphi}_{\nu} * M\right)(n-1) \geq 0$ that $u(t) \geq v(t)$. The proof is complete.

Theorem 3.4. Assume that the assumptions in Theorem 3.2 are satisfied, except replacing ${ }_{a} \Delta_{h, *}^{\beta}$ by ${ }_{a} \Delta_{h}^{\beta}$. Then we have $\|x(a+n h)\| \leq\left[\frac{\bar{V}(0)}{\alpha_{1}} E_{(\beta)}\left(-\frac{\alpha_{3}}{\alpha_{2}} h^{\beta}, n\right)\right]^{\frac{1}{a}}$, where $x(a+n h)$ is the solution of the system (3.2).

Proof. It follows from Lemma 3.4 and $\bar{V}(n) \geq 0$ for $n \in \mathbb{N}_{0}$ that $\left({ }_{a} \Delta_{h, *}^{\beta} \bar{V}\right)(n) \leq$ $\left({ }_{a} \Delta_{h}^{\beta} \bar{V}\right)(n) \leq-\alpha_{3}\|x\|^{a b}$. Following the same proof in Theorem 3.2, we get $\| x(a+$ $n h) \| \leq\left[\frac{\bar{V}(0)}{\alpha_{1}} E_{(\beta)}\left(-\frac{\alpha_{3}}{\alpha_{2}} h^{\beta}, n\right)\right]^{\frac{1}{a}}$. The proof is complete.

Theorem 3.5. Assume that $x=0$ is an equilibrium point of the system (3.2), let $\mathcal{D} \subseteq \mathbb{R}^{n}$ be a domain containing the origin, and there exist a function $V: \mathbb{N}_{0} \times \mathcal{D} \rightarrow \mathbb{R}$, and class $\mathcal{K}$-function $\alpha$ satisfying

$$
\begin{aligned}
& \bar{V}(n) \geq \alpha(\|x\|), \\
& \left({ }_{0} \Delta_{h}^{\beta} \bar{V}\right)(n) \leq 0,
\end{aligned}
$$

where $n \in \mathbb{N}_{0}, x \in \mathcal{D}, \beta \in(0,1)$. Then the equilibrium point $x=0$ is stable.

Proof. It follows from Lemma 3.5, and the inequality (3.21) that $\bar{V}(n) \leq \bar{V}(0)$. Again by inequality (3.20), we have

$$
\|x\| \leq \alpha^{-1}(\bar{V}(0))=\alpha^{-1}\left(V\left(0, x_{0}\right)\right) .
$$

According to the definition of stability, the equilibrium point $x=0$ is stable. The proof is complete. 


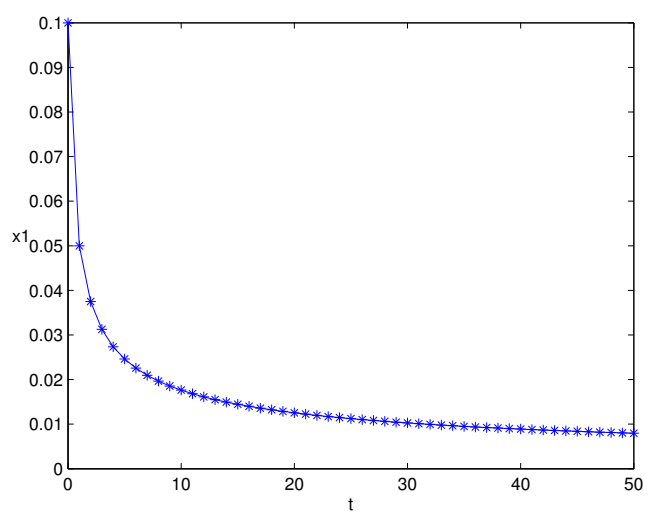

Figure 4.1: Asymptotic stability of $x_{1}$ for $\nu=0.5$.

\section{NUMERICAL RESULTS}

Now, we give two numerical examples to illustrate the application of the results established in the previous section.

Example 4.1. Consider the following fractional order $h$-difference systems

$$
\left\{\begin{array}{l}
\left({ }_{a} \Delta_{h, *}^{\nu} x_{1}\right)(t)=-x_{1}(t+\nu h)+x_{2}^{5}(t+\nu h), \quad x_{1}(a)=0.1, \\
\left({ }_{a} \Delta_{h, *}^{\nu} x_{2}\right)(t)=-x_{2}(t+\nu h)-\frac{1}{3} x_{1}(t+\nu h), \quad x_{2}(a)=0.2,
\end{array}\right.
$$

where $\nu=0.5, a=-0.5, h=1, t \in(h \mathbb{N})_{0}$, and this difference system has a trivial solution $x(t)=\left(x_{1}(t), x_{2}(t)\right)^{T}=0$.

We define the following positive definite Lyapunov function

$$
V\left(x_{1}(t), x_{2}(t)\right)=x_{1}^{2}(t)+x_{2}^{2}(t) .
$$

Then the function $\bar{V}(n)=V\left(n, x_{1}(n-0.5), x_{2}(n-0.5)\right)$ satisfies the assumptions of Theorem 3.2, so the trivial solution of the considered system is Mittag-Leffler stable, as a consequence, by Lemma 3.2, the origin of the system (4.1) is asymptotically stable, as it can be seen from Figures 4.1 and 4.2 .

The comparison of the norm of the solution to the system (4.1) with the MittagLeffler function $E_{(0.5)}(-0.2, n)$ multiplied by $\left\|\left(x_{1}(a), x_{2}(a)\right)\right\|=\sqrt{0.05}$ is presented in Figure 4.3 for $n=0,1, \cdots, 50$. The solution of the system (4.1) satisfies the following inequality

$$
\|(x(a+n h), y(a+n h))\|=\sqrt{(x(a+n h))^{2}+(y(a+n h))^{2}} \leq \sqrt{0.05} E_{(0.5)}(-0.2, n) .
$$




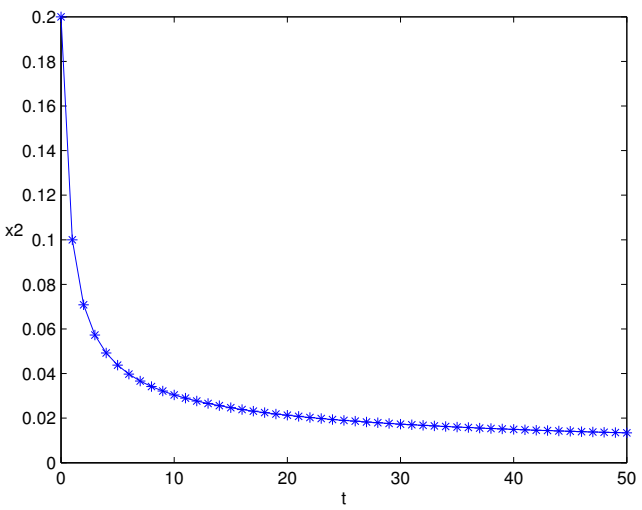

Figure 4.2: Asymptotic stability of $x_{2}$ for $\nu=0.5$.

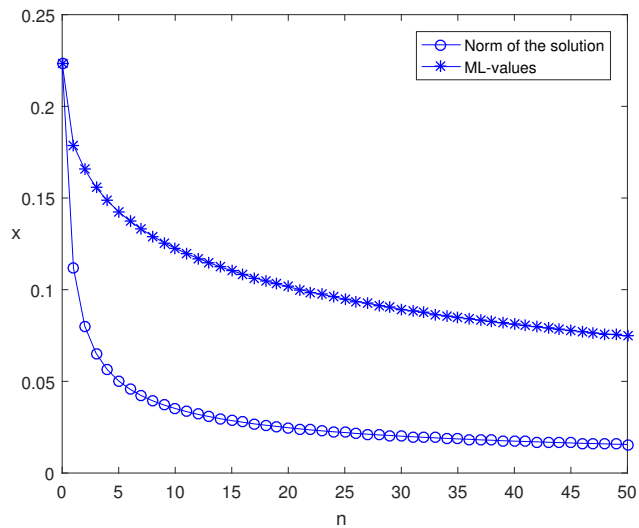

Figure 4.3: The comparison of the norm of the solution to the system (4.1) with $\sqrt{0.05} E_{(0.5)}(-0.2, n)$ for $n=0,1, \cdots, 50$.

Example 4.2. Consider the following fractional order $h$-difference systems

$$
\left\{\begin{array}{l}
\left({ }_{a} \Delta_{h}^{\nu} x_{1}\right)(t)=-\frac{1}{10} x_{1}(t+\nu h)-\frac{1}{10} x_{2}^{9}(t+\nu h), \quad x_{1}(a)=0.1, \\
\left({ }_{a} \Delta_{h}^{\nu} x_{2}\right)(t)=\frac{1}{10} x_{1}^{9}(t+\nu h)-\frac{1}{10} x_{2}(t+\nu h), \quad x_{2}(a)=0.2,
\end{array}\right.
$$

where $\nu=0.5, a=-0.5, h=1, t \in(h \mathbb{N})_{0}$, and this difference system has a trivial solution $x(t)=\left(x_{1}(t), x_{2}(t)\right)^{T}=0$.

We define the following positive definite Lyapunov function

$$
V\left(x_{1}(t), x_{2}(t)\right)=x_{1}^{10}(t)+x_{2}^{10}(t) .
$$

Then the function $\bar{V}(n)=V\left(n, x_{1}(n-0.5), x_{2}(n-0.5)\right)$ satisfies the assumptions of Theorem 3.4, so the trivial solution of the considered system is Mittag-Leffler stable, 


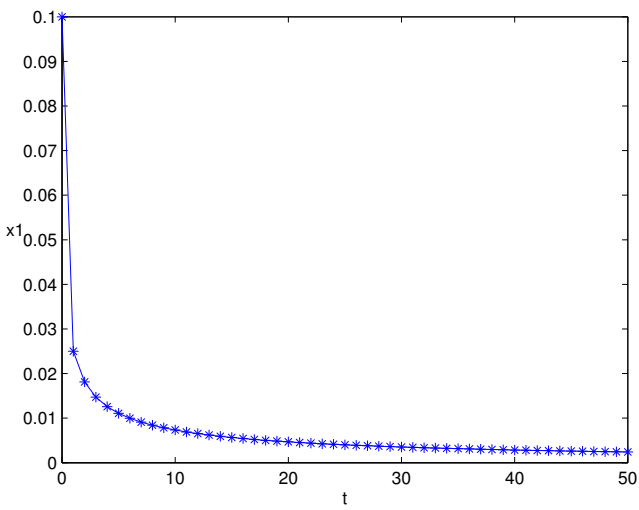

Figure 4.4: Asymptotic stability of $x_{1}$ for $\nu=0.5$.

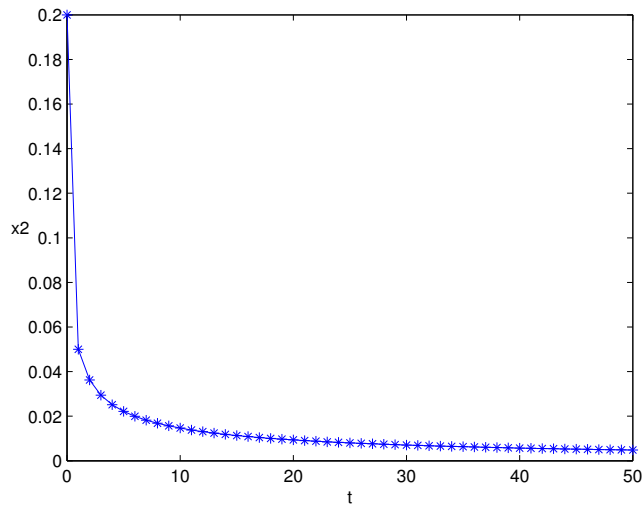

Figure 4.5: Asymptotic stability of $x_{2}$ for $\nu=0.5$.

consequently, from Lemma 3.2, the origin of the system (4.2) is asymptotically stable, as it can be seen from Figures 4.4 and 4.5.

The comparison of the norm of the solution to the system (4.2) with the MittagLeffler function $E_{(0.5)}(-0.2, n)$ multiplied by $\left\|\left(x_{1}(a), x_{2}(a)\right)\right\|=\sqrt{0.05}$ is presented in Figure 4.6 for $n=0,1, \cdots, 50$. The solution of the system (4.2) satisfies the following inequality

$$
\|(x(a+n h), y(a+n h))\|=\sqrt{(x(a+n h))^{2}+(y(a+n h))^{2}} \leq \sqrt{0.05} E_{(0.5)}(-0.2, n) .
$$




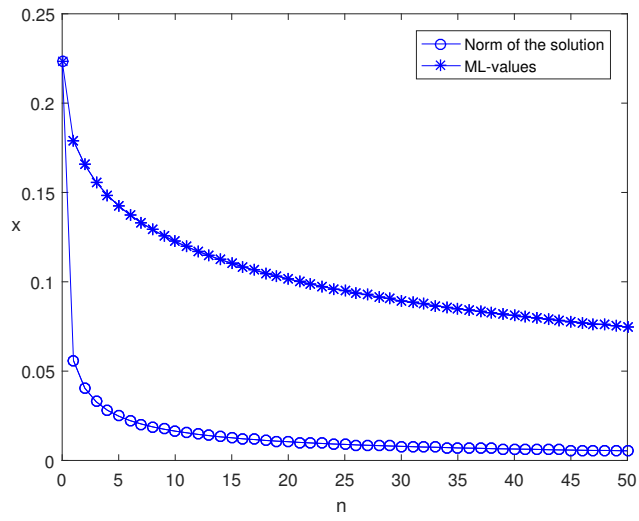

Figure 4.6: The comparison of the norm of the solution to the system (4.2) with $\sqrt{0.05} E_{(0.5)}(-0.2, n)$ for $n=0,1, \cdots, 50$.

\section{CONCLUSION}

In the above parts, we studied the stability of the fractional order $h$-difference systems. The definition of Mittag-Leffler stability was introduced, and the sufficient conditions for Mittag-Leffler stability were presented by extending the Lyapunov direct method to such systems. Furthermore, by weakening the restriction on Lyapunov function, we proved the stability for those systems. Finally, two numerical examples are given to show the established results.

\section{ACKNOWLEDGMENTS}

This paper is supported by the National Natural Science Foundation of China (112713 80), Guangdong Province Key Laboratory of Computational Science, and the International Program for Ph.D. Candidates, Sun Yat-Sen University.

\section{REFERENCES}

[1] A. Kilbas, H. Srivastava, J. Trujillo, Theory and applications of fractional differential equations. Elsevier, 2016.

[2] I. Podlubny, Fractional Differential Equations. Mathematics in Sciences and Engineering, Academic Press, San Diego, 1999.

[3] T. Kaczorek, Selected Problems of Fractional Systems Theory. Springer-Verlag, Berlin, Heidelberg, 2011. 
[4] K. S. Miller, B. Ross, Fractional difference calculus. In: Proc. International Symposium on Univalent Functions, Fractional Calculus and their Applications, Nihon University, Kōriyama 1988, pp. 139-152.

[5] D. Mozyrska, E. Girejko, Overview of fractional $h$-difference operators. In: Advances in Harmonic Analysis and Operator Theory: The Stefan Samko Anniversary Volume (A. Almeida, L. Castro, F. O. Speak, eds.), Operator Theory: Advances and Applications, Springer 2013, pp. 253-268. DOI:10.1007/978-3-03480516-2_14

[6] M. Wyrwas, E. Girejko, D. Mozyrska, E. Pawluszewicz, Stability of fractional difference systems with two orders. In: Advances in the Theory and Applications of Non-integer Order Systems (W. Mitkowski, J. Kacprzyk, and J. Baranowski, eds.), Lect. Notes Electr. Engrg. 257, Springer International Publishing, Switzerland 2013, pp. 41-52. DOI:10.1007/978-3-319-00933-9_4

[7] B. G. Jia, X. Liu, F. F. Du, M. Wang, The solution of a new Caputo-like fractional h-difference equation, Rocky Mountain J. Math. 2017, to appear.

[8] M. Wyrwas, E. Pawluszewicz, E. Girejko, Stability of nolinear $h$-difference systems with $n$ fractional orders. Kybernetika, 51 (1) (2015), 112-136.

[9] D. Mozyrska, E. Girejko, M. Wyrwas, Comparison of $h$-difference fractional operators. In: Advances in the Theory and Applications of Non-integer Order Systems (W. Mitkowski, J. Kacprzyk, and J. Baranowski, eds.), Lect. Notes Electr. Engrg. 257, Springer International Publishing, Switzerland 2013, pp. 191-197. DOI:10.1007/978-3-319-00933-9_17

[10] N. R. O. Bastos, R. A. C. Ferreira, D. F. M. Torres, Discrete time fractional variational problems, Signal Processing, 91 (2011), 513-524. DOI: 10.1016/j.sigpro.201 0.05 .001

[11] D. Mozyrska, M. Wyrwas, The $\mathcal{Z}$-transform method and delta type fractional difference operators. Discret. Dyn. Nat. Soc., 2015 (2015), Article ID 852734, 12 pages.

[12] S. Momani, S. Hadid, Lyapunov stability solutions of fractional integrodifferential equations. Int. J. Math. Math. Sci., 47 (2004), 2503-2507.

[13] L. G. Zhang, J. M. Li, G. P. Chen, Extension of Lyapunov second method by fractional calculus. Pure Appl. Math., 3 (2005), 1008-5513.

[14] V. E. Tarasov, Fractional stability, 2007. Available online: http://arxiv.org/abs/ $0711.2117 \mathrm{v} 1$.

[15] D. Matignon, Stability properties for generalized fractional differential systems. ESAIM Proc., 5 (1998), 145-158. 
[16] Y. Li, Y. Q. Chen, I. Podlubny, Mittag-Leffler stability of fractional order nonlinear dynamic systems. Automatica, 45 (2009), 1965-1969. DOI:10.1140/epjst/e20 11-01379-1

[17] Y. Li, Y. Q. chen, I. Podlubny, Stability of fractional-order nonlinear dynamic systems: Lyapunov direct method and generalized Mittag-Leffler stability. Comput. Math. Appl., 59 (2010), 1810-1821. DOI:10.1016/j.camwa.2009.08.019

[18] J. M. Yu, H. Hu, S. B. Zhou, X. R. Lin, Generalized Mittag-Lefler stability of multi-variables fractional order nonlinear systems. Automatica, 49 (2013), 1798-1803.

[19] F. R. Zhang, C. P. Li, Y. Q. Chen, Asymptotical stability of nolinear fractional differential systems with Caputo derivative. Internat. J. Differ. Equ., Volume 2011, Article ID 635165, 12 pages.

[20] M. Wyrwas, D. Mozyrska, On Mittag-Leffler stability of fractional order difference systems. In: Advances in Modeling and Control of Non-integer order systems, (K. J. Latawiec et al. eds.), Lect. Notes Electr. Engrg. 320, Springer International Publishing, Switzerland 2015, pp. 209-220. DOI:10.1007/978-3319-09900-2_19

[21] F. Jarad, T. Abdeljawad, D. Baleanu, K. Biçen, On the stability of some discrete fractional nonautonomous systems. Abstr. Appl. Anal., Volume 2012, Article ID 476581, 9 pages. doi:10.1155/2012/476581 